\title{
Cost-Effectiveness of SGLT2is Versus DPP4is Among Type 2 Diabetes Patients with and Without Established Cardiovascular Disease: A Model-Based Simulation Analysis Using 10-Year Real-World Data and Systematic Review
}

\author{
Zi-Yang Peng \\ National Cheng Kung University \\ Chun-Ting Yang \\ National Cheng Kung University \\ Huang-Tz Ou ( $\nabla$ huangtz@mail.ncku.edu.tw ) \\ National Cheng Kung University \\ Shihchen Kuo \\ University of Michigan Medical School
}

\section{Research Article}

Keywords: cost-effectiveness analysis, model-based, simulation study, real-world data, SGLT2is, DPP4is

Posted Date: December 2nd, 2021

DOI: https://doi.org/10.21203/rs.3.rs-1126975/v1

License: (1) (i) This work is licensed under a Creative Commons Attribution 4.0 International License. Read Full License 


\section{Abstract}

Background: We conducted a model-based economic analysis of sodium-glucose cotransporter-2 inhibitors (SGLT2is) versus dipeptidyl peptidase-4 inhibitors (DPP4is) in type 2 diabetes (T2D) patients with and without established cardiovascular disease (CVD) using 10-year real-world data.

Methods: A Markov model was utilized to estimate healthcare costs and quality-adjusted life-years (QALYs) over a 10-year simulation time horizon from a healthcare sector perspective, with both costs and QALYs discounted at $3 \%$ annually. Model inputs were derived from analyses of Taiwan's National Health Insurance Research Database or published studies of Taiwanese populations. The primary outcome measure was the incremental cost-effectiveness ratios (ICERs). Incorporated with our study findings, a structured systematic review was conducted to synthesize updated evidence on the cost-effectiveness of SGLT2is versus DPP4is.

Results: Over 10 years, use of SGLT2is versus DPP4is yielded ICERs of $\$ 3,244$ and $\$ 4,186$ per QALY gained for T2D patients with and without established CVD, respectively. Results were robust across a series of sensitivity and scenario analyses, showing ICERs between $\$-1,074$ (cost-saving) and \$8,467 per QALY gained for T2D patients with established CVD and between $\$ 369$ and $\$ 37,122$ per QALY gained for T2D patients without established CVD. A systematic review revealed a cost-effective or even cost-saving profile of using SGLT2is for T2D treatment.

Conclusions: Use of SGLT2is versus DPP4is was highly cost-effective for T2D patients regardless of patients' CVD history in real-world clinical practice. Our results extend current evidence by demonstrating SGLT2is as an economically rational alternative over DPP4is for T2D treatment in routine care. Future research is warranted to explore heterogenous economic benefits of SGLT2is given diverse patient characteristics in clinical settings.

\section{Background}

A substantial burden attributable to cardiovascular diseases (CVDs) among patients with type 2 diabetes (T2D) remains a major challenge for healthcare systems globally $(1,2)$. Sodium-glucose cotransporter-2 inhibitors (SGLT2is), originally developed as glucose-lowering agents (GLAs), have shown beneficial effects on the cardiovascular system $(3,4)$. Compared with dipeptidyl peptidase-4 inhibitors (DPP4is), which are widely used as add-on oral GLAs for T2D patients who failed metformin therapy (5-8), the use of SGLT2is substantially reduces the risks of CVDs and mortality (9).

A growing body of evidence on the cost-effectiveness analysis (CEA) of SGLT2is versus DPP4is suggests that the use of SGLT2is is cost-effective or even cost-saving compared with DPP4is (10-18). However, research gaps remain due to the marked heterogeneity of treatment experiences in different regions and the data or analysis limitations of existing studies. Most of these studies adopted treatment efficacy data from clinical trials with limited short-term follow-ups to project long-term outcomes in model-based simulation economic analyses. The applicability of results from these CEA studies, which were based on selective and homogeneous patient populations in clinical trials, to diverse patient populations in the real world remains unclear. Furthermore, considering that health utility and healthcare cost estimates could be sensitive to study 
regions, settings and populations (e.g., countries and healthcare systems), the CEA results using health utility and cost data not specific to the given study settings/populations may limit generalizability to inform local clinical care and health policy decisions $(12-14,18)$. Moreover, given differences in the disease progression of T2D between patients with and without pre-existing CVDs, which were not differentiated in previous CEA studies $(10-15,17,18)$, the results remain inconclusive to inform personalized medicine on tailoring a treatment to patients' CVD risks.

Against this background, we conducted an economic analysis study using a model-based simulation analysis integrated with 10-year real-world data to evaluate the cost-effectiveness of SGLT2is versus DPP4is in T2D patients from a healthcare sector perspective in Taiwan. We employed population- and settingspecific model inputs to enhance the generalizability of study results to usual clinical practice settings in Taiwan, and stratified analyses based on patients' CVD history to provide informative evidence for individualized treatment decisions. Moreover, a structured systematic review was conducted to incorporate our study findings for providing the updated evidence on the cost-effectiveness of using SGLT2is versus DPP4is for T2D patients.

\section{Methods}

\section{Base-case model structure and assumptions}

A state-transition Markov model was used to project the progression of CVDs among T2D patients and simulate the effects of using SGLT2is versus DPP4is on health and economic outcomes. The model comprised five health states: T2D without any CVD events, heart failure (HF), myocardial infarction (MI), stroke, and all-cause death (Additional file 1). A hypothetical cohort was assumed for the modeling analyses based on patient characteristics reported in a previous Taiwanese study (19): T2D patients with a diabetes duration of 8 years and the initiation of SGLT2is or DPP4is at 55 years old. Patients could progress from T2D without any CVD events to other health states in a yearly cycle over a 10-year simulation horizon. Quality-adjusted life-years (QALYs) and healthcare costs were rewarded in each cycle and discounted at 3\% annually over the simulation horizon. All analyses, including model inputs (i.e., transition probability, health utility, and cost parameters) and economic evaluations, were conducted for T2D patients stratified by CVD history.

\section{Transition probabilities between health states}

To reflect the real-world effectiveness of SGLT2is versus DPP4is in Taiwan, transition probabilities between modeled health states were derived from Taiwan's National Health Insurance Research Database (NHIRD) and the published literature on the Taiwanese population (20). Specifically, in the DPP4i group, T2D patients who newly initiated DPP4is in 2010 were identified from the NHIRD and followed until death or the end of the database (i.e., December 31, 2018), whichever came first, to estimate CVD risks in each follow-up year as the model inputs for transition probabilities of progressing from T2D without any CVD events to HF, MI, and stroke. Regarding transition probabilities from other health states to death, the mortality estimates were obtained from a risk score system that has been established for the prediction of CVD-specific and all-cause 
mortalities in Taiwanese T2D patients as a function of patient socio-demographics, lifestyle behaviors, and diabetes-related clinical characteristics (e.g., treatments and biomarkers) (20).

In the SGLT2i group, the comparative treatment effects (i.e., relative hazards) of SGLT2is versus DPP4is on risks of CVDs and death were incorporated with the transition probabilities of the DPP4i group to convert the transition probabilities of the SGLT2i group using the ProbToProb function in TreeAge. The relative hazards were estimated using Cox proportional hazard models for analyses of the propensity score-matched pairs of new SGLT2i or DPP4i users identified in 2017 from the NHIRD and followed from the initiation of study drugs until the occurrence of study outcomes (i.e., HF, MI, stroke, and death) or the end of the database (i.e., December 31, 2018), whichever came first (19). Estimation procedures and the data of transition probabilities for each model pathway between health states are detailed in Additional file 2-4.

\section{Health utility and healthcare costs}

The health utility parameters in the model were based on a population-based study that estimated the mean health utility score of Taiwanese patients with T2D along with health utility penalties attributable to patient demographic and clinical characteristics (21). Specifically, the health utility inputs were estimated by subtracting the utility penalties of given patient characteristics from the mean health utility score of T2D patients. For example, a base-case patient without CVD history (baseline health utility: 0.983 ) who progressed to HF (health utility penalty of 0.237 ) had a health utility score of $0.746(0.983-0.237)$.

The cost parameters associated with different health states were based on a population-based study of Taiwanese T2D patients that estimated the mean annual healthcare cost of T2D patients, and cost multipliers associated with patient demographic and clinical characteristics (2). Healthcare costs for a given health state in the model were estimated by multiplying the mean annual healthcare cost with cost multipliers of patient characteristics associated with that health state. For example, for a base-case patient without CVD history (baseline healthcare cost: USD 349) who had a diabetes duration of 8 years (cost multiplier: 0.85), initiated a DPP4i or SGLT2i (cost multiplier: 1.78), and progressed to HF (cost multiplier: 2.24), the estimated healthcare cost input was USD 1,183 (USD $349 \times 0.85 \times 1.78 \times 2.24$ ). Moreover, the drug cost of SGLT2is (or DPP4is) was estimated as the average drug cost of all available SGLT2is (or DPP4is) reimbursed by Taiwan's National Health Insurance (NHI) program in 2017 (22). Costs were converted to 2020 values using the medical component of the consumer price index in Taiwan and are presented in USD (23). The health utility and cost input parameters used in the modeling analyses are detailed in Additional file 5.

\section{Base-case analysis}

The total QALYs and healthcare costs of a patient in each treatment group were simulated over a 10-year time horizon from a healthcare sector perspective. The 10-year cost-effectiveness of SGLT2i and DPP4i treatments was then measured in terms of the incremental cost-effectiveness ratios (ICERs), calculated as incremental total healthcare costs divided by incremental total QALYs. As recommended by the World Health Organization (24), the willingness-to-pay (WTP) thresholds in this economic analysis study were set as one and three times the per capita gross domestic product (GDP) of Taiwan in 2020, which were USD 30,038 and USD 90,114 (25), respectively, to determine whether the use of SGLT2is versus DPP4is was highly cost- 
effective (i.e., USD 0 < ICER $\leq$ USD 30,038) or cost-effective (i.e., USD 30,038 < ICER $\leq$ USD 90,114). The impact inventory for the components considered in the cost-effectiveness analyses is provided in Additional file 6.

\section{Sensitivity analyses}

Deterministic sensitivity analyses (DSAs) and probabilistic sensitivity analyses (PSAs) were conducted to quantify the impact of parameter uncertainties on ICER estimates. In the DSAs, the lower and upper bounds of each model input (i.e., the ranges shown in Additional file 3-5) were applied, and the key drivers of ICERs were identified as the parameters whose variations yielded a change of over $15 \%$ in ICER estimates, and then illustrated in a tornado diagram. The PSAs were performed using a Monte Carlo simulation with 10,000 iterations, in which all model parameter inputs simultaneously varied in the plausible ranges with pre-defined distributions (Additional file 3-5) that were determined by the characteristics of the parameters and their levels of certainty.

\section{Scenario analyses}

Several scenario analyses were conducted to confirm the robustness of the study findings. First, the simulation time horizon was extended to 20 and 30 years and shortened to 1, 3, and 5 years to assess the uncertainty that arises from the length of the simulation period. Second, in the base-case analysis, the baseline demographics and laboratory data of modeled subjects that mirrored the average or common values of the characteristics of the Taiwanese T2D population (as shown in Additional file 2) were used in the risk score system (20) to estimate the mortality risk, which contributed 0 points in the risk score calculation. This assumption might thus underestimate the mortality of our modeled subjects. Therefore, a scenario analysis was conducted to use higher mortalities predicted by a risk score system that assumed the risk factor values to be above the average levels of T2D patients. Third, CVD risks (i.e., pathways a, b, and c) were varied with a range of $\pm 25 \%$ to account for disease progression rates that may change with the aging of the patient cohort or the evolution of clinical management over time. Fourth, considering potentially unmeasured confounding effects on the estimates of the relative hazards associated with SGLT2is versus DPP4is in claims-based research (19), the hazard ratios from a network meta-analysis of clinical trials were adopted in a sensitivity analysis. Fifth, a break-even cost analysis was conducted, where the drug cost of SGLT2is was varied by $10 \%$ to $50 \%$ of the base-case value against the ICERs to demonstrate the results of adjusting reimbursement prices for SGLT2is in Taiwan's NHI program.

Analyses of the NHIRD were performed using SAS 9.4 (SAS Institute Inc, Cary, NC) and the model-based economic analyses were performed using TreeAge Pro 2020 decision analysis software (TreeAge Software, LLC, Williamstown, MA).

\section{Systematic review}

Two authors (Peng and Yang) independently searched for studies that reported the cost-effectiveness of SGLT2is versus DPP4is in PubMed and Embase from the inception of each database until July 2, 2021. Details of the search strategy and the key terms are provided in Additional file 7. Only original research 
articles with an available full text published in English were included in the review process. All authors screened the eligibility of each study. Any disagreements were resolved by group discussion and consensus. A flowchart of the study selection is shown in Additional file 8. The base-case ICER values of the included studies were adjusted to 2020 values using the country-specific consumer price index and then converted to USD from the country-specific currency using an annual currency exchange rate (26). Further, to provide an overview of economic analysis results of SGLT2is versus DPP4is, a figure was plotted to summarize the ICERs of the present and published studies included in the systematic review. The figure shows the median, lowest, and highest ICER values for all SGLT2is and each individual SGLT2i against the WTP thresholds in Taiwan and the commonly used cost-effective (CE) threshold in the US (27) to determine whether using SGLT2is versus DPP4is is: 1) cost-saving (ICER < 0), 2) highly cost-effective (ICER $\leq$ USD 25,000 [CE threshold in the US] or USD 30,038 [Taiwan's per capita GDP]), 3) cost-effective (USD 25,000 < ICER $\leq$ USD 50,000 [CE threshold in the US] or USD 30,038 < ICER $\leq$ USD 90,114 [between one and three times Taiwan's per capita GDP]), 4) marginally cost-effective (USD 50,000 < ICER $\leq$ USD 100,000 [CE threshold in the US]), or 5) not cost-effective (ICER > USD 100,000 [CE threshold in the US] or USD 90,114 [three times Taiwan's per capita GDP]).

\section{Results}

\section{Base-case analyses}

Over a 10-year simulation, HF was the most common CVD among T2D patients with CVD history and stroke was the most common CVD among T2D patients without CVD history (as shown in Figure 1). Compared with DPP4is, the use of SGLT2is yielded 0.198 QALYs gained at an additional cost of USD 644 (ICER: USD 3,244 per QALY gained) for T2D patients with CVD history, and 0.245 QALYs gained at an additional cost of USD 1,025 (ICER: USD 4,185 per QALY gained) for T2D patients without CVD history (Table 1).

\section{Sensitivity analyses}

The DSA results in Figure 2 show that the top two influential drivers for ICER values were the annual drug costs of DPP4is and the hazard ratio of SGLT2is versus DPP4is on HF for T2D patients with CVD history, and the annual drug costs of DPP4is and the hazard ratio of SGLT2is versus DPP4is on all-cause death for those without CVD history. The PSA results show that the use of SGLT2is versus DPP4is for T2D, regardless of patients' baseline CVD history, is considered highly cost-effective in $85 \%-100 \%$ of the model iterations at the WTP threshold of USD 30,038 based on base-case and scenario analyses, except for the 1-year simulation of T2D patients without CVD history (Table 1 and Figure 3 ).

\section{Scenario analyses}

The results of the scenario analyses are generally consistent with those of the base-case analyses, showing highly cost-effective for using SGLT2i versus DPP4is, except for the result of the analysis based on a 1-year simulation of T2D patients without CVD history (Table 1). The results of break-even analyses show that using SGLT2i versus DPP4i would yield more QALYs with a lower total healthcare cost (i.e., cost-saving 
treatment) when the annual drug cost of SGLT2i is lower than USD 304 for T2D patients with CVD history and USD 257 for those without CVD history (Additional file 9).

\section{Systematic review}

Nine articles were included in the systematic review of CEAs of SGLT2i versus DPP4i use in T2D populations. All of them utilized existing valid simulation models (e.g., Cardiff) with individual-based modeling approaches to project the progression of T2D. As shown in Additional file 10, all studies adopted effectiveness data from clinical trials, and six of them applied country-specific health utility parameters. Only one study performed a subgroup analysis on the CEA stratified by previous CVD history. The study results across these studies were consistent (10-18), showing that SGLT2is versus DPP4is is cost-effective (11-13, 15-17) or even cost-saving $(10,14,18)$ given a WTP threshold from the US or Taiwan. The ICERs from the systematic review findings and the present study results are summarized in Additional file 11.

\section{Discussion}

This economic analysis applied real-world and population-specific data as model inputs to reflect the costeffectiveness of SGLT2is versus DPP4is for T2D in usual clinical practice settings in Taiwan. The present study findings, together with the systematic review results, provide supportive evidence that promotes the use of SGLT2is as an economically reasonable alternative to DPP4is for T2D patients in real-world clinical practice regardless of baseline patient status of CVDs.

\section{Comparison with current evidence on the cost-effectiveness of SGLT2is versus DPP4is}

The conclusions for this cohort-based modeling CEA are generally consistent with existing studies that primarily utilized individual-based modeling approaches to evaluate the long-term health and economic outcomes of SGLT2is versus DPP4is (Additional file 10). Specifically, the cost-effectiveness results of SGLT2is versus DPP4is observed in our study are comparable with those in the literature, showing that the use of SGLT2is is a cost-effective $(11-13,15-17)$ or even cost-saving $(10,14,18)$ treatment option compared with DPP4is for T2D. Our study further extends current evidence by demonstrating SGLT2is as an economically rational alternative to DPP4is for T2D treatment in routine care. In addition, the probabilities of being cost-effective for using SGLT2is versus DPP4is obtained from the PSAs (i.e., 100\% for patients with and without CVD history) in this study are similar to the probability estimates reported in the literature (i.e., near $(10,15,17)$ or equal to $100 \%(14,16))$. However, direct comparisons of cost-effectiveness estimate between our study and previous studies should be done with caution given the differences in the study populations and settings, follow-up periods, analytical design, approaches, and assumptions.

Additionally, several influential drivers for ICER values found in this study (Figure 2) have been identified in previous studies $(16,17)$, including drug costs of DPP4is and SGLT2is $(16,17)$, treatment effects of SGLT2is versus DPP4is on cardiovascular and mortality outcomes $(16)$, and health utilities of health states $(16,17)$. DPP4i drug cost was the most prominent driver of ICERs in our study regardless of patients' status of CVD history. This may be explained by a wide range of drug costs of DPP4is (i.e., USD 87 to USD 319 per year in Taiwan) used as model inputs under Taiwan's health insurance system. DPP4is have been reimbursed by 
Taiwan's NHI program since 2006. The drug reimbursement fees of DPP4is have changed considerably owing to the launch of generic DPP4i drugs, adjustments of drug pricing, and changes in relevant reimbursement policies under the Taiwan NHI Administration's regulations (28). Although variations in DPP4i drug costs had a great impact on ICER values, the cost-effective result of using SGLT2is versus DPP4is remained robust in our sensitivity analyses (Figure 2). Regarding other drivers of ICERs, some heterogeneity between the study patients with and without established CVD, described below, should be acknowledged.

Comparison of dominant variables for base-case ICERs between T2D patients with and without established $\underline{\mathrm{CVD}}$

For the base-case ICER, the treatment effects (i.e., hazard ratios) of SGLT2is versus DPP4is on HF, stroke, and all-cause death were the dominant drivers, other than DPP4i drug costs, for T2D patients with established CVDs, followed by utility penalties of CVDs, drug costs of SGLT2is, and discount rate. However, for T2D patients without established CVDs, only the treatment effects of SGLT2is versus DPP4is on stroke and all-cause death and drug costs of SGLT2is affected the base-case ICER by more than $15 \%$.

As shown in Figure 1, there are some discrepancies in the composition of the estimated event rates of CVDs and all-cause death between the two simulated cohorts (T2D patients with and without CVD history). HF and all-cause death were the two most common events among T2D patients with CVD history in our analyses. Given the apparent benefits of SGLT2i use on HF and all-cause death (9), it is expected that CEA results would be affected by the hazard ratios of SGLT2is versus DPP4is on these two outcomes for T2D patients with CVD history. In contrast, the risk of developing HF was relatively low among T2D patients without CVD history, while stroke and all-cause death accounted for most clinical events for this population. As a result, the hazard ratios of SGLT2is versus DPP4is on stroke and all-cause death, instead of HF, were identified as the leading drivers for the ICER in T2D patients without CVD history. This means that the treatment benefits of SGLT2is versus DPP4is on CVDs and death would be more evident when the patients' baseline risks of these clinical events were higher, which would further affect the CEA results. In addition, the utility penalties associated with $\mathrm{MI}, \mathrm{HF}$, and stroke had considerable impacts on the ICER for patients with CVD history but not those without CVD history. This may also be explained by the relatively low CVD risks in patients without CVD history, and thus the QALYs gained contributed by SGLT2i-associated cardiovascular benefits were trivial in this population.

The DSA findings suggest that among T2D patients with established CVDs whose cardiovascular risks are considerably higher than those without established CVDs, the economic benefit of SGLT2is versus DPP4is would mainly come from the effectiveness of SGLT2is in lowering risks of CVD events (including HF, MI, and stroke) and all-cause death that subsequently lead to improved QALYs and reduced healthcare costs of patients. Conversely, the less beneficial effects of SGLT2is versus DPP4is on CVD events, particularly HF and MI risks, in T2D patients without CVD history might not meaningfully dominate the CEA results, and in this case, the drug costs of SGLT2is are one of the leading drivers of the ICER.

Clinical and health policy implications 
Based on our study findings, the use of SGLT2is in real-world practice should be encouraged owing to its lower risks of major clinical events and economic benefits compared with DPP4is. Moreover, from the perspective of the healthcare sector, this study provides supporting evidence for using SGLT2is to facilitate the cost-effective allocation of healthcare resources. Specifically, Additional file 9, which shows the ICERs of SGLT2is versus DPP4is against various SGLT2i drug costs, could assist health policy-makers in pricing SGLT2i reimbursement fees. For example, SGLT2is become a cost-saving treatment option compared with DPP4is when the SGLT2i drug costs are lower than USD 304 and USD 257 per year for patients with established CVD and those without CVD history, respectively. These break-even points may serve as a reference for the reimbursement pricing adjustment of SGLT2is. In addition, it is expected that the economic benefit of SGLT2is would be more amplified when the cost of SGLT2is is significantly reduced owing to the launch of their generic drugs.

Differences in the cost-effectiveness of SGLT2is versus DPP4is contributed by patients' CVD history were revealed in this study, suggesting the importance for prioritizing the treatment for subgroup populations who can benefit more from using SGLT2is clinically and economically. For example, we found that the use of SGLT2is yielded a more favorable cost-effectiveness profile among patients with established CVDs than those without CVD history; i.e., the ICER estimates were consistently lower in patients with CVD versus those without CVD history in base-case analyses and most scenario analyses (Table 1). Therefore, given limited healthcare resources, the reimbursement or incentive health policies could be tailored to prioritize the use of SGLT2is in T2D populations with CVD history. Further research is warranted to identify the subgroup patient populations with specific characteristics (e.g., comorbid chronic kidney disease, elderly patients) who can benefit more from SGLT2i use to optimize healthcare resource allocation.

\section{Strengths and limitations}

Our economic evaluation attentively applied several study designs that can reflect real-life scenarios, including 1) derivation of effectiveness parameters (i.e., transition probabilities regarding CVD events) from a large-scale, real-world T2D cohort population, 2) incorporation of time-varying transition probabilities (i.e., annual risks of CVDs, increased mortalities with aging) in modeling analyses, and 3) stratification of the analyses for patients with established CVD and those without established CVD; such a stratification analysis was less often considered in previous CEAs. In addition, to ensure the applicability of the study results in Taiwan's healthcare setting, we used data from population-based studies that analyzed Taiwanese T2D populations for the effectiveness, health utility, and cost parameter inputs in the model (i.e., Chen et al.'s study (2), Kuo et al.'s study (21), and the T2D cohort identified from the NHIRD for costs, utility, and effectiveness parameters (19), respectively). The model inputs derived from the same target population (i.e., the T2D cohort from NHIRD) in our study minimize the uncertainties of CEA results that arise from the heterogeneity of multiple data sources.

Several limitations of our study should be acknowledged. First, our Markov decision model included only health states of CVDs and death without considering other clinical outcomes such as kidney diseases and adverse effects of treatments. Given growing evidence about SGLT2i-associated renal benefits $(29,30)$, the cost-effectiveness of SGLT2is versus DPP4is revealed in the present study could be underestimated. In 
addition, the impact of adverse drug effects on the CEA results might be negligible given the low incidence of adverse effects (e.g., severe hypoglycemia, diabetic ketoacidosis). Second, the analyses were not performed from the societal perspective because costs from informal healthcare and non-healthcare sectors were not included due to data unavailability. Lastly, the improved health-related quality of life associated with the use of SGLT2is other than those contributed by reduced CVD and mortality events were not considered, e.g., the SGLT2i-associated benefit of body weight loss on patients' health-related quality of life (31). We might thus have underestimated the economic value of SGLT2is.

\section{Conclusions}

In summary, SGLT2i versus DPP4i use for real-world T2D patients regardless of CVD history in Taiwan is highly cost-effective. Future research is encouraged to explore the subgroup of patients with specific characteristics regarding their health and economic benefits obtained from SGLT2is to facilitate clinical care and health policy decisions and optimize healthcare resource allocation.

\section{Abbreviations}

CVDs: cardiovascular diseases

T2D: type 2 diabetes

SGLT2is: sodium-glucose cotransporter-2 inhibitors

GLAs: glucose-lowering agents

DPP4is: dipeptidyl peptidase-4 inhibitors

CEA: cost-effectiveness analysis

HF: heart failure

Ml: myocardial infarction

QALYs: quality-adjusted life-years

NHIRD: National Health Insurance Research Database

NHI: National Health Insurance

ICERs: incremental cost-effectiveness ratios

WTP: willingness-to-pay

GDP: gross domestic product

DSAs: deterministic sensitivity analyses 
PSAs: probabilistic sensitivity analyses

CE: cost-effective

\section{Declarations}

\section{Ethical approval and consent to participate}

This study was approved by the Institutional Review Board of National Cheng Kung University (A-EX-109035).

\section{Consent for publication}

Not applicable.

\section{Availability of data and materials}

Data sharing is not applicable to this study as data management and analysis were only allowed to be conducted in Health and Welfare Data Science Center in Taiwan for data privacy and safety concerns.

\section{Competing interests}

All authors declare no competing interests regarding this work.

\section{Funding}

This project was supported by grants from the Ministry of Science and Technology in Taiwan (grant MOST 109-2320-B-006 -047-MY3) (Huang-Tz Ou). The funder had no role in the design and conduct of the study; collection, management, analysis, and interpretation of the data; preparation, review or approval of the manuscript; and decision to submit the manuscript for publication.

\section{Authors' contributions}

Z.Y.P. designed the study, performed the literature review, analyzed, and interpreted the data, and wrote the manuscript. C.T.Y. designed the study, performed the literature review, analyzed, and interpreted the data, and wrote the manuscript. H.T.O. provided study materials, designed the study, interpreted the data, and wrote the manuscript. S.K. designed the study, interpreted the data, and reviewed/edited the manuscript. All authors approved the final manuscript.

\section{Acknowledgment}

We are grateful to Health Data Science Center, National Cheng Kung University Hospital, for providing administrative and technical support.

\section{References}


1. American Diabetes Association. Economic Costs of Diabetes in the U.S. in 2017. Diabetes Care. 2018;41(5):917-928.

2. Chen HY, Kuo S, Su PF, Wu JS, Ou HT. Health Care Costs Associated With Macrovascular, Microvascular, and Metabolic Complications of Type 2 Diabetes Across Time: Estimates From a Population-Based Cohort of More Than 0.8 Million Individuals With Up to 15 Years of Follow-up. Diabetes Care. 2020;43(8):1732-1740.

3. Salah HM, Al'Aref SJ, Khan MS, Al-Hawwas M, Vallurupalli S, Mehta JL, Mounsey JP, Greene SJ, McGuire DK, Lopes RD, Fudim M. Effect of sodium-glucose cotransporter 2 inhibitors on cardiovascular and kidney outcomes-Systematic review and meta-analysis of randomized placebo-controlled trials. Am Heart J. 2021;232:10-22.

4. Zelniker TA, Wiviott SD, Raz I, Im K, Goodrich EL, Bonaca MP, Mosenzon O, Kato ET, Cahn A, Furtado RHM, Bhatt DL, Leiter LA, McGuire DK, Wilding JPH, Sabatine MS. SGLT2 inhibitors for primary and secondary prevention of cardiovascular and renal outcomes in type 2 diabetes: a systematic review and meta-analysis of cardiovascular outcome trials. Lancet. 2019;393(10166):31-39.

5. Wilkinson S, Douglas I, Stirnadel-Farrant H, Fogarty D, Pokrajac A, Smeeth L, Tomlinson L. Changing use of antidiabetic drugs in the UK: trends in prescribing 2000-2017. BMJ Open. 2018;8(7):e022768.

6. McCoy RG, Dykhoff HJ, Sangaralingham L, Ross JS, Karaca-Mandic P, Montori VM, Shah ND. Adoption of New Glucose-Lowering Medications in the U.S.-The Case of SGLT2 Inhibitors: Nationwide Cohort Study. Diabetes Technol Ther. 2019;21(12):702-712.

7. Kim J, Park S, Kim H, Je NK. National trends in metformin-based combination therapy of oral hypoglycaemic agents for type 2 diabetes mellitus. Eur J Clin Pharmacol. 2019;75(12):1723-1730.

8. Kameda T, Kumamaru H, Nishimura S, Kohsaka S, Miyata H. Use of oral antidiabetic drugs in Japanese working-age patients with type 2 diabetes mellitus: dosing pattern for metformin initiators. Curr Med Res Opin. 2020;36(5):749-756.

9. Kohsaka S, Lam CSP, Kim DJ, Cavender MA, Norhammar A, Jørgensen ME, Birkeland KI, Holl RW, Franch-Nadal J, Tangri N, Shaw JE, Ilomäki J, Karasik A, Goh SY, Chiang CE, Thuresson M, Chen H, Wittbrodt E, Bodegård J, Surmont F, Fenici P, Kosiborod M; CVD-REAL 2 Investigators and Study Group. Risk of cardiovascular events and death associated with initiation of SGLT2 inhibitors compared with DPP-4 inhibitors: an analysis from the CVD-REAL 2 multinational cohort study. Lancet Diabetes Endocrinol 2020;8;606-15.

10. Sabapathy S, Neslusan C, Yoong K, Teschemaker A, Johansen P, Willis M. Cost- effectiveness of Canagliflozin versus Sitagliptin When Added to Metformin and Sulfonylurea in Type 2 Diabetes in Canada. J Popul Ther Clin Pharmacol. 2016;23(2):e151-168.

11. Charokopou M, McEwan P, Lister S, Callan L, Bergenheim K, Tolley K, Postema R, Townsend R, Roudaut M. Cost-effectiveness of dapagliflozin versus DPP-4 inhibitors as an add-on to Metformin in the Treatment of Type 2 Diabetes Mellitus from a UK Healthcare System Perspective. BMC Health Serv Res. 2015;15:496.

12. Neslusan C, Teschemaker A, Johansen P, Willis M, Valencia-Mendoza A, Puig A. ost-Effectiveness of Canagliflozin versus Sitagliptin as Add-on to Metformin in Patients with Type 2 Diabetes Mellitus in 
Mexico. Value Health Reg Issues. 2015;8:8-19.

13. Tzanetakos C, Tentolouris N, Kourlaba G, Maniadakis N. Cost-Effectiveness of Dapagliflozin as Add-On to Metformin for the Treatment of Type 2 Diabetes Mellitus in Greece. Clin Drug Investig. 2016;36(8):649-659.

14. Chakravarty A, Rastogi M, Dhankhar P, Bell KF. Comparison of costs and outcomes of dapagliflozin with other glucose-lowering therapy classes added to metformin using a short-term cost-effectiveness model in the US setting. J Med Econ. 2018;21(5):497-509.

15. Ramos M, Foos V, Ustyugova A, Hau N, Gandhi P, Lamotte M. Cost-Effectiveness Analysis of Empagliflozin in Comparison to Sitagliptin and Saxagliptin Based on Cardiovascular Outcome Trials in Patients with Type 2 Diabetes and Established Cardiovascular Disease. Diabetes Ther. 2019;10(6):21532167.

16. Reifsnider O, Kansal A, Pimple P, Aponte-Ribero V, Brand S, Shetty S. Cost- effectiveness analysis of empagliflozin versus sitagliptin as second-line therapy for treatment in patients with type 2 diabetes in the United States. Diabetes Obes Metab. 2021;23(3):791-799.

17. Hu S, Deng X, Ma Y, Li Z, Wang Y, Wang Y. Cost-Utility Analysis of Dapagliflozin Versus Saxagliptin Treatment as Monotherapy or Combination Therapy as Add-on to Metformin for Treating Type 2 Diabetes Mellitus. Appl Health Econ Health Policy. 2021;19(1):69-79.

18. Van der Linden N, Van Olst S, Nekeman S, Uyl-de Groot CA. The cost-effectiveness of dapagliflozin compared to DPP-4 inhibitors in the treatment of type 2 diabetes mellitus in the Netherlands. Diabet Med. 2021;38(4):e14371.

19. Yang CT, Peng ZY, Chen YC, Ou HT, Kuo S. Cardiovascular benefits with favorable renal, amputation and hypoglycemic outcomes of SGLT-2 inhibitors in type 2 diabetes from the Asian perspective: A population-based cohort study and systematic review. (under review by Diabetes Research and Clinical Practice).

20. Liu CS, Li Cl, Wang MC, Yang SY, Li TC, Lin CC. Building clinical risk score systems for predicting the allcause and expanded cardiovascular-specific mortality of patients with type 2 diabetes. Diabetes Obes Metab. 2021;23(2):467-479.

21. Kuo S, Yang CT, Chen HY, Ou HT. Valuing health states of people with type 2 diabetes: Analyses of the nationwide representative linked databases. J Diabetes Investig. 2021 Sep;12(9):1749-1758.

22. Taiwanese National Health Insurance Administration (NHIA), Ministry of Health and Welfare. List of reimbursed drugs by NHI. [Internet] Available from https://www.nhi.gov.tw/QueryN/Query1.aspx on July $4^{\text {th }}, 2021$.

23. Taiwanese National Statistics. Consumer price index. [Internet] Available from https://statdb.dgbas.gov.tw/pxweb/Dialog/price.asp?mp=4 on December 2020.

24. Bertram MY, Lauer JA, De Joncheere K, Edejer T, Hutubessy R, Kieny MP, Hill SR. Cost-effectiveness thresholds: pros and cons. Bull World Health Organ. 2016;94(12):925-930.

25. Taiwanese National Statistics. Latest Indicators. [Internet] Available from https://www.stat.gov.tw/point.asp?index=1 on December 2020. 
26. Exchange Rates Organization. [Internet] Available from https://www.exchangerates.org.uk/ on Sep $24^{\text {th }}$, 2021.

27. Siegel KR, Ali MK, Zhou X, Ng BP, Jawanda S, Proia K, Zhang X, Gregg EW, Albright AL, Zhang P. Costeffectiveness of Interventions to Manage Diabetes: Has the Evidence Changed Since 2008? Diabetes Care. 2020;43(7):1557-1592.

28. Hsu JC, Lu CY. The evolution of Taiwan's National Health Insurance drug reimbursement scheme. Daru. 2015;23(1):15.

29. Seino Y, Kim DJ, Yabe D, Tan EC, Chung WJ, Ha KH, Nangaku M, Node K, Klement R, Yasui A, Lei WY, Lee S, Kyaw MH, Deruaz-Luyet A, Brodovicz KG, H-H Sheu W; EMPRISE East Asia study group. Cardiovascular and renal effectiveness of empagliflozin in routine care in East Asia: Results from the EMPRISE East Asia study. Endocrinol Diabetes Metab 2021;4:e00183.

30. Idris I, Zhang R, Mamza JB, Ford M, Morris T, Banerjee A, Khunti K. Lower risk of hospitalization for heart failure, kidney disease and death with sodium-glucose co-transporter- 2 inhibitors compared with dipeptidyl peptidase-4 inhibitors in type 2 diabetes regardless of prior cardiovascular or kidney disease: A retrospective cohort study in UK primary care. Diabetes Obes Metab. 2021;23(10):2207-2214.

31. Ishii H, Nakajima H, Kamei N, Niiya T, Hiyoshi T, Hiramori Y, Ohtsu S, Noto T, Shimono D. Quality-of-Life Comparison of Dapagliflozin Versus Dipeptidyl Peptidase 4 Inhibitors in Patients with Type 2 Diabetes Mellitus: A Randomized Controlled Trial (J-BOND Study). Diabetes Ther. 2020;11(12):2959-2977.

\section{Tables}

Table 1. Results of cost-effectiveness of SGLT2is versus DPP4is in base-case analysis and scenario analyses 


\begin{tabular}{|c|c|c|c|c|c|c|c|}
\hline \multicolumn{3}{|l|}{ QALYs } & \multicolumn{3}{|c|}{ Costs (USD) } & \multirow{2}{*}{$\begin{array}{l}\text { ICER } \\
\text { (USD per } \\
\text { QALY } \\
\text { gained) }\end{array}$} & \multirow{2}{*}{$\begin{array}{l}\text { Probability } \\
\text { of being } \\
\text { highly } \\
\text { cost- } \\
\text { effective* } \\
\text { for } \\
\text { SGLT2is } \\
\text { vS. DPP4is } \\
\text { in PSA }\end{array}$} \\
\hline SGLT2is & DPP4is & $\begin{array}{l}\text { Incre- } \\
\text { mental }\end{array}$ & SGLT2is & DPP4is & $\begin{array}{l}\text { Incre- } \\
\text { mental }\end{array}$ & & \\
\hline
\end{tabular}

\section{Base-case analyses (10-year simulation)}

\begin{tabular}{lllllllll}
$\begin{array}{l}\text { With CVD } \\
\text { history }\end{array}$ & 6.492 & 6.294 & 0.198 & 11,306 & 10,661 & 644 & $3,244.07$ & $100.0 \%$ \\
$\begin{array}{l}\text { Without } \\
\begin{array}{l}\text { CVD } \\
\text { history }\end{array}\end{array}$ & 8.154 & 7.909 & 0.245 & 9,147 & 8,122 & 1,025 & $4,185.64$ & $100.0 \%$ \\
\hline
\end{tabular}

\section{Scenario analyses}

With CVD history

\begin{tabular}{|c|c|c|c|c|c|c|c|c|}
\hline $\begin{array}{l}\text { Mortality } \\
\text { considering } \\
\text { diabetes- } \\
\text { related risk } \\
\text { factors } \\
\text { with values } \\
\text { over } \\
\text { average } \\
\text { levels }^{\dagger}\end{array}$ & 2.519 & 1.685 & 0.834 & 14,733 & 14,252 & 480 & 576.47 & $100.0 \%$ \\
\hline
\end{tabular}

$\begin{array}{lllllllll}\text { Elevated } & 6.448 & 6.245 & 0.203 & 11,598 & 11,007 & 591 & 2,908.77 & 100.0 \% \\ \text { CVD risks } & & & & & & & & \\ \text { in DPP4i } & & & & & & & & \\ \text { group (25\% } & & & & & & & \\ \text { increase) } & & & & & & \end{array}$

$\begin{array}{lllllllll}\text { Decreased } & 6.540 & 6.348 & 0.191 & 10,991 & 10,270 & 720 & 3,770.20 & 100.0 \% \\ \text { CVD risks } & & & & & & & & \\ \text { in DPP4i } & & & & & & & \\ \text { group (25\% } & & & & & & & \\ \text { reduction) } & & & & & & \end{array}$

\begin{tabular}{|c|c|c|c|c|c|c|c|c|}
\hline $\begin{array}{l}\text { Treatment } \\
\text { effects of } \\
\text { SGLT2is } \\
\text { from NMA } \\
\text { of clinical } \\
\text { trials }\end{array}$ & 6.421 & 6.294 & 0.127 & 11,480 & 10,662 & 818 & $6,439.57$ & $100.0 \%$ \\
\hline
\end{tabular}

$\begin{array}{lcccccccc}\begin{array}{l}\text { 1-year } \\ \text { simulation }\end{array} & 0.782 & 0.778 & 0.003 & 1,238 & 1,251 & 23 & 7,659.27 & 85.6 \% \\ \begin{array}{l}\text { 3-year } \\ \text { simulation }\end{array} & 2.247 & 2.221 & 0.026 & 3,684 & 3,574 & 109 & 4,281.99 & 99.8 \% \\ \begin{array}{l}5-\text {-year } \\ \text { simulation }\end{array} & 3.591 & 3.527 & 0.064 & 5.991 & 5.748 & 243 & 3,797.91 & 100.0 \%\end{array}$




\begin{tabular}{|c|c|c|c|c|c|c|c|c|}
\hline $\begin{array}{l}\text { 20-year } \\
\text { simulation }\end{array}$ & 10.579 & 10.021 & 0.557 & 20,423 & 18,918 & 1,505 & $2,700.90$ & $100.0 \%$ \\
\hline $\begin{array}{l}\text { 30-year } \\
\text { simulation }\end{array}$ & 12.710 & 11.745 & 0.965 & 27,335 & 24,883 & 2,452 & $2,541.73$ & $100.0 \%$ \\
\hline \multicolumn{9}{|c|}{ Without CVD history } \\
\hline $\begin{array}{l}\text { Mortality } \\
\text { considering } \\
\text { diabetes- } \\
\text { related risk } \\
\text { factors } \\
\text { with values } \\
\text { over } \\
\text { average } \\
\text { levels }^{\dagger}\end{array}$ & 3.613 & 2.369 & 1.243 & 11,655 & 11,196 & 459 & 369.08 & $100.0 \%$ \\
\hline $\begin{array}{l}\text { Elevated } \\
\text { CVD risks } \\
\text { in DPP4i } \\
\text { group (25\% } \\
\text { increase) }\end{array}$ & 8.115 & 7.848 & 0.267 & 9,226 & 8,246 & 980 & $3,664.73$ & $100.0 \%$ \\
\hline $\begin{array}{l}\text { Decreased } \\
\text { CVD risks } \\
\text { in DPP4i } \\
\text { group (25\% } \\
\text { reduction) }\end{array}$ & 8.193 & 7.972 & 0.220 & 9,068 & 7,993 & 1,074 & $4,864.24$ & $100.0 \%$ \\
\hline $\begin{array}{l}\text { Treatment } \\
\text { effects of } \\
\text { SGLT2is } \\
\text { from NMA } \\
\text { of clinical } \\
\text { trials }\end{array}$ & 8.022 & 7.909 & 0.113 & 9,355 & 8,122 & 1,232 & $10,907.14$ & $100.0 \%$ \\
\hline $\begin{array}{l}\text { 1-year } \\
\text { simulation }\end{array}$ & 0.964 & 0.961 & 0.003 & 976 & 860 & 115 & $37,122.45$ & $27.9 \%$ \\
\hline $\begin{array}{l}\text { 3-year } \\
\text { simulation }\end{array}$ & 2.785 & 2.758 & 0.027 & 2,887 & 2,552 & 335 & $12,548.61$ & $100.0 \%$ \\
\hline $\begin{array}{l}\text { 5-year } \\
\text { simulation }\end{array}$ & 4.471 & 4.401 & 0.070 & 4,743 & 4,199 & 543 & $7,753.02$ & $100.0 \%$ \\
\hline $\begin{array}{l}\text { 20-year } \\
\text { simulation }\end{array}$ & 13.518 & 12.734 & 0.748 & 16,989 & 15,085 & 1,903 & $2,427.23$ & $100.0 \%$ \\
\hline $\begin{array}{l}\text { 30-year } \\
\text { simulation }\end{array}$ & 16.632 & 15.155 & 1.478 & 23,350 & 20,485 & 2,865 & $1,938.86$ & $100.0 \%$ \\
\hline
\end{tabular}

Abbreviations: SGLT2is, sodium-glucose cotransporter-2 inhibitors; DPP4is, dipeptidyl peptidase 4 inhibitors; QALY, quality-adjusted life-year; PSA, probabilistic sensitivity analysis; USD, United States dollar; ICER, incremental cost-effectiveness ratio; CVD, cardiovascular disease; NMA, network meta-analysis.

*The willingness-to-pay threshold was set as one time the per capita gross domestic product in Taiwan in 2020 (i.e., USD 30,038). 
${ }^{+} \mathrm{A}$ 14-point risk score that comprised the following risk factors and score points (with a range of 0 to 5 , indicating no to high impact on patient's mortality) was applied to predict the mortality of study cohort patients in the scenario analysis: 55 years old ( 3 points), diabetes duration of 8 years ( 2 points), glucoselowering agents ( 1 point), smoking behavior ( 1 point), education $\leq 5$ years ( 2 points), body mass index < $18.5 \mathrm{~kg} / \mathrm{m}^{2}$ (1 point), variation of fast plasma glucose $\geq 22.3 \%$ (1 point), variation of glycated hemoglobin $\geq 4.5 \%$ ( 1 point), variation of diastolic blood pressure $\geq 5.5 \%$ ( 1 point), triglycerides $\geq 150 \mathrm{mg} / \mathrm{dL}$ ( 1 point), and presence of peripheral neuropathy (1 point).

\section{Figures}

(a)

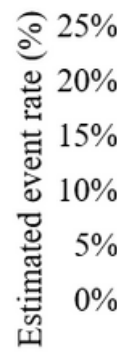

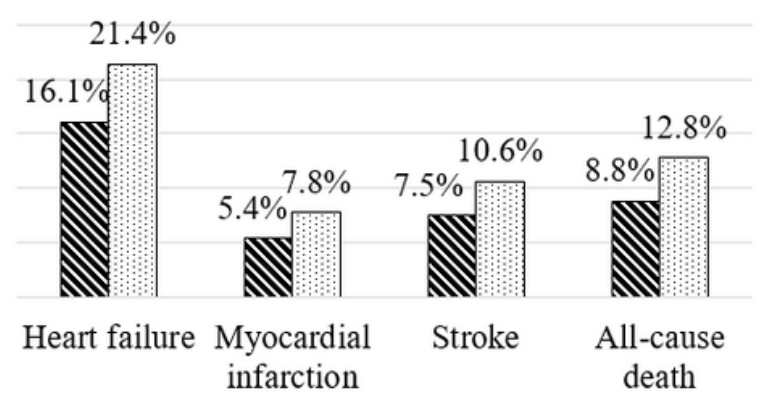

\$ SGLT2i users ? DPP4i users (b)

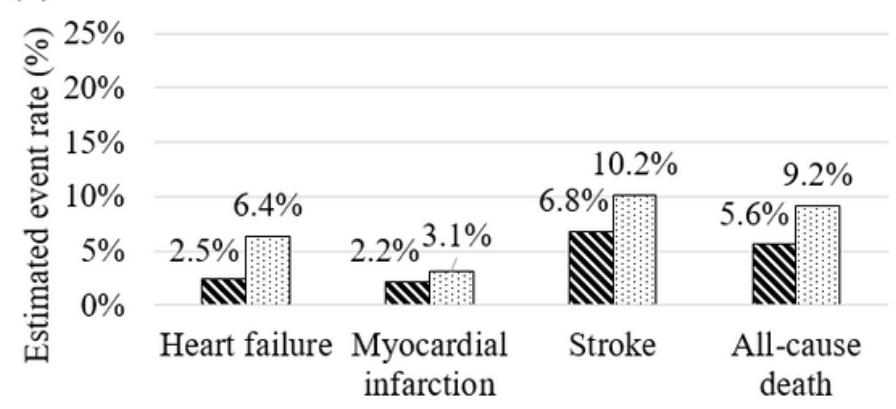

\$SGLT2i users $\quad$ DDPP4i users

\section{Figure 1}

Estimated event rates of cardiovascular diseases and all-cause death over 10-year simulation horizon in patients (a) with and (b) without cardiovascular disease history Abbreviations: SGLT2is, sodium-glucose cotransporter-2 inhibitors; DPP4is, dipeptidyl peptidase 4 inhibitors.

\section{Figure 2}

Tornado diagram of deterministic sensitivity analyses results in type 2 diabetes patients (a) with and (b) without cardiovascular disease history Abbreviations: DPP4is, dipeptidyl peptidase 4 inhibitors; USD, United States dollar; HR, hazard ratio; HF, heart failure; MI, myocardial infarction; SGLT2is, sodium-glucose cotransporter-2 inhibitors; ICER, incremental cost-effectiveness ratio; QALY, quality-adjusted life-year. *The hazard ratios refer to the estimated relative risk of SGLT2is versus DPP4is on CVDs or death. Note: Only the parameters whose variations (i.e., lower and upper bounds) changed the ICER values by $\geq 15 \%$ are presented. 


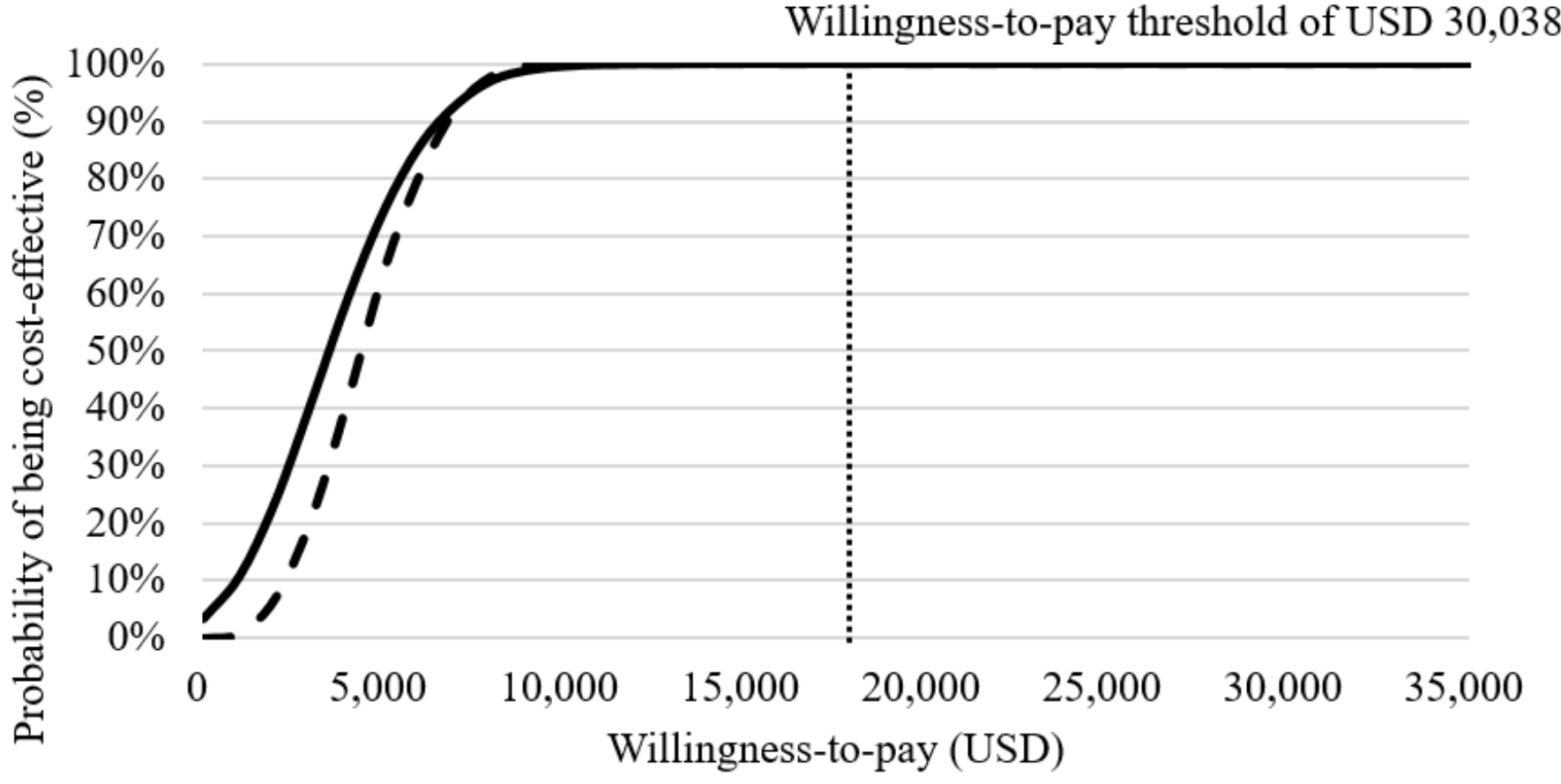

- - T2D patients without CVD history —T2D patients with CVD history

\section{Figure 3}

Cost-effectiveness acceptability curve of SGLT2is versus DPP4is in type 2 diabetes patients with and without cardiovascular disease history in base-case analyses Abbreviations: CVD, cardiovascular disease; T2D, type 2 diabetes; USD, United States dollar.

\section{Supplementary Files}

This is a list of supplementary files associated with this preprint. Click to download.

- Additionalfile1.pdf

- Additionalfile2.pdf

- Additionalfile3.pdf

- Additionalfile4.pdf

- Additionalfile5.pdf

- Additionalfile6.pdf

- Additionalfile7.pdf

- Additionalfile9.pdf

- Additionalfile11.pdf 